\title{
Aligning Interferograms from Single-baseline InSAR by Local Frequency Estimation
}

\author{
Jiao Guo*, Shenglan Wang and Baofeng Su \\ College of Mechanical and Electronic Engineering \\ Northwest A \& F University \\ Yangling, China \\ *Corresponding author
}

\begin{abstract}
It is feasible to obtain multibaseline InSAR data with repeat passes of existing single-baseline InSAR systems. Due to high coherence, the single-pass pair of SAR images can be accurately aligned using existing methods. However, there exist great difficulties in alignment of multiple-pass SAR images. Fortunately, repeat-pass interferograms are corresponding to the same terrain, i.e., DEM (Digital Elevation Model). Hence the interferograms are coherent, even if the SAR images are decorrelated completely. From this point, this paper proposes an innovative strategy to align the interferograms rather than the SAR images. Since the interferograms generated by different effective baseline are not identical, this paper proposes a new method to align interferograms by local frequency estimation. Finally, the computer simulation results show that the proposed method can align interferograms accurately.
\end{abstract}

Keywords-synthetic aperture radar interferometry(InSAR); Digital Elevation Model(DEM); multibaseline; local frequency estimation

\section{INTRODUCTION}

Synthetic aperture radar interferometry (InSAR) is one of the most important remote sensing techniques to acquire terrain digital elevation models (DEMs) [1][2]. SAR interferometry illuminates the same scene to obtain two or more SAR images from different incident angles at the same or different time, i.e., single-pass or multiple-pass mode. And the phases of the SAR image pair are processed in interferometric way to determine the height of each scattering element. Finally, the DEMs are generated. This advanced remote sensing technique, which can work either day or night and through cloud cover, has numerous applications in the fields of topography, geomorphology, seismology, etc [1]-[3].

To our knowledge, single-pass spaceborne InSAR systems (for instance, the Shuttle Radar Topography Mission (SRTM) and the German Distributed Satellites Tandem-X and TandemL) and airborne InSAR systems are composed of only two antennae, that is, only two antenna phase centers are installed to receive the transmitted electromagnetic wave backscattered from the same scene simultaneously. This conventional singlebaseline InSAR system with two antenna phase centers has been proved to have the capability to provide DEMs of high quality for general terrains. However, for complicated topographies containing highly sloping regions or discontinuous surfaces (such as man-made building, canyons and steep mountains that exist numerously on the Earth), the performance decreases severely due to the serious undersampling phases and layover phenomena. In the past decade, the concept of multibaseline InSAR systems (with three or even more antenna phase centers) has been widely exploited to overcome the drawbacks associated with conventional single-baseline InSAR systems [4]-[9].

Unfortunately, single-pass multibaseline InSAR systems have not been used extensively because of the high cost. Meanwhile, multibaseline interferometric data acquired by multiple passes are not able to achieve results of high quality because of the serious temporal decorrelation. Hence utilizing single-pass or multiple-pass data separately results in respective drawbacks. In practice, it is feasible to acquire multibaseline interferometric data through repeat passes of a single-baseline InSAR system. Therefore, it is practical to combine single-pass and multiple-pass InSAR with the aim of constructing multibaseline InSAR. For instance, the German Distributed Satellites Tandem- $X$ is designed to possess at most four acquisitions with different baseline lengths in order to obtain DEM for hilly and mountainous areas [10].

As is well known, accurate alignment of SAR images is the indispensable precondition of multibaseline interferometric processing. For instance, the multibaseline two-dimensional phase unwrapping methods such as the CRT method [8] and the Maximum Likelihood [9] both require precise alignment of each pixel in the SAR image pair. The pair of SAR images produced by single-baseline interferometric SAR can be conveniently aligned by conventional methods [11][12] due to the high coherence. However, the serious temporal and spatial baseline decorrelation result in extreme difficulties in the alignment operation between multiple-pass SAR images, especially in the case of large effective cross-track baseline lengths. In this paper, we propose an innovative approach to accurately align multiple-pass SAR images of a single-baseline InSAR. The basic idea is that each interferogram is figured out from each pass of a single-baseline InSAR and the multiple interferograms are then aligned, which can avoid the influence of temporal and spatial baseline decorrelation induced by multiple passes. In other words, the alignment operation is performed for the multiple interferograms rather than the SAR images. The interferograms, which are corresponding to the terrain elevation fluctuations, are correlated, even if the multiple-pass SAR images are decorrelated completely. For the interferograms generated by different baseline lengths are different, the proposed method exploits the local frequency 
estimates of interferograms to complete the alignment. This paper only deals with the alignment of two-pass SAR image pairs, since it can be easily extended to the multiple-pass case.

\section{PRINCIPLE OF INTERFEROGRAM ALIGNMENT}

The geometry of two passes of a single-baseline InSAR system is shown in Figure. I, where the two passes are represented by Pass1 and Pass2, the corresponding antenna phase centers are represented by $\left(A_{11}, A_{12}\right)$ and $\left(A_{21}, A_{22}\right)$, and $b_{1}$ and $b_{2}$ are the cross-track baseline lengths of the two passes, respectively. The imaging reference plane illustrated in Figure. I is assumed to be zero-Doppler plane. The axis $\mathrm{Y}$ and $\mathrm{Z}$ denote the ground range and height directions, respectively. The point $P$ is the target. The interferometric data acquired by the two passes are processed via conventional procedures (including pre-filtering, SAR imaging, SAR image coregistration) separately in order to generate two interferograms; that is, the two interferograms corresponding to two passes are firstly acquired. The effective cross-track baseline length of Pass1 differs from that of Pass2 owing to the changes of viewing angles or antenna configuration for airborne InSARs or the formation-flying distances for distributed satellite InSARs, which is also the effective way to construct multibaseline InSAR. Consequently, the two interferograms corresponding to two different effective crosstrack baseline lengths are different, and straightforward interferogram alignment is theoretically impossible. The most straightforward way is to apply phase unwrapping operation to the interferograms separately with the purpose of obtaining two DEMs, and then the correlation of the two DEMs are performed to complete the interferogram alignment. However, it is necessary to pass through the key step of two-dimensional phase unwrapping, which is known as the most difficult operation in InSAR data processing procedures [13].

As mentioned above, the different interferograms generated by multiple passes with different effective crosstrack baselines are still corresponding to the identical terrain. From the view of this point, this paper proposes a new method for aligning the multi-pass interferograms based on the topography variation. For the two-pass interferograms corresponding to the different baseline lengths, we try to find out the coherent information and the local frequency (i.e., topography variation) is considered as such a bridge to connect the two interferograms. In order to achieve robust estimates of topography variation for each pixel in the interferogram, we make the following assumption that the terrain in the local rectangular window can be approximated by a slant plane with a constant range slope and a constant azimuth slope [14]. In other words, the two-dimensional complex signals in the local window are composed of only single two-dimensional frequency component. Consequently, the parameter estimation technique can be adopted to obtain robust estimation for the topography variation of each pixel. From the approximate relation between the phase and the height of the terrain, we can get the following equation,

$$
h(m, n)=a_{i} ?\left[j_{i}(m, n) \quad k_{i}(m, n) ? 2 p\right]
$$

where $m$ and $n$ denote the azimuth and range coordinates, respectively, the subscript $i$ is used to distinguish the two passes ( $i=1,2), h(m, n)$ and $j_{i}(m, n)$ are the height and the ambiguous phase of the pixel $(m, n)$, respectively, $k_{i}(m, n)$ $\left(k_{i}(m, n)=0\right.$, 北, 2...) is the integer ambiguities of the phase of pixel $(m, n), a_{i}$ is the scaling factor which transforms the phase to height and can be approximated as,

$$
a_{i} » \frac{l_{i} R_{i} \sin q_{i}}{4 p B_{\wedge i}}
$$

where $l_{i}$ is the wavelength of the carrier, $R_{i}$ is the reference slant range, $q_{i}$ is the incident angle, and $B_{\wedge i}$ is the effective cross-track baseline length.

(1) and (2) reveal the fact that the terrain height of SAR pixel is approximatively proportional with the interferometric phase. The topography variations (i.e., range slope and azimuth slope according to the assumption) are the product of the local frequency of the complex signals and the scaling factor which can be approximated as a constant. Consequently, the local frequencies of the multiple interferograms generated by different cross-track baselines can be exploited to align the interferogram pair.

According to the assumed conditions, the following equation holds,

$$
\varphi(m+p, n+q)+K(m+p, n+q) \cdot 2 \pi=p \cdot f_{a}+q \cdot f_{r}+\varphi(m, n)
$$

where $\left(f_{a}, f_{r}\right)$ denote the azimuth frequency and range frequency to be determined, respectively, $K(m+p, n+q)$ is the integer ambiguities of the pixel $(m+p, n+q)$ relative to the current estimating pixel with the coordinates $(m, n)$, the symbols $p$ and $q$ denote the azimuth and range distance of the pixels in the local window relative to the central pixel $(m, n)$.

To acquire the estimates of the parameters $\left(f_{a}, f_{r}\right)$, there exist several methods available such as maximum likelihood and MUSIC instantaneous frequency estimation [15]. In this paper, we adopt the optimizing method to estimate the local frequencies and the cost function is defined as what follows,

$$
\begin{aligned}
\left(\hat{f}_{a}, \hat{f}_{r}\right) & =\max \sum_{p=-P}^{P} \sum_{q=-Q}^{Q} \mid s_{1}(m+p, n+q) \\
& \cdot s_{2}{ }^{*}(m+p, n+q) \exp \left(j\left(-2 \pi p f_{a}-2 \pi q f_{r}\right)\right) \mid
\end{aligned}
$$

where $s_{1}$ and $s_{2}$ are the interferometric SAR image pair and the size of the local window is $(2 P+1) \times(2 Q+1)$. The estimated frequencies of the pixel $(m, n)$ can be computed by 
solving the (4). However, solving (4) is a two-dimensional optimizing problem and there exist heavy computation load. We decompose the one two-dimensional cost function to two one-dimensional ones to reduce the computation load, thus estimating the range frequency and azimuth frequency separately.

From the terrain model of slant plane (3), we can conclude that the azimuth frequency for each range position is identical in the local window, so the azimuth frequency can be determined by the following equation,

$$
\begin{aligned}
\hat{f}_{a}= & \max \sum_{p=-P}^{P} \mid \sum_{q=-Q}^{Q} s_{1}(m+p, n+q) \\
& \cdot s_{2}{ }^{*}(m+p, n+q) \exp \left(-j 2 \pi p f_{a}\right) \mid
\end{aligned}
$$

Similarly, we can determine the range frequency by

$$
\begin{aligned}
& \hat{f}_{r}= \max \sum_{q=-Q}^{Q} \mid \sum_{p=-P}^{P} s_{1}(m+p, n+q) \\
& \cdot s_{2}{ }^{*}(m+p, n+q) \exp \left(-j 2 \pi q f_{r}\right) \mid
\end{aligned}
$$

From (5) and (6), we can obtain the local azimuth and range frequency estimation for each pixel in the interferogram. The cross-correlation algorithm to the local frequency is applied to determine the azimuth and range shift amounts, and then the interferograms are aligned according to the shift amounts. It should be emphasized that the azimuth and range shifts are sensitive to azimuth frequency and range frequency. Therefore, the cross-correlations of the azimuth and range local frequency are employed to obtain the shift amounts in the azimuth and range directions, respectively.

\section{Performance InVESTigation}

This section investigates the performance of proposed method with simulated data.

The repeat passes of spaceborne single-baseline InSAR system are assumed with the effective cross-track baseline lengths (two-way) of $834.2 \mathrm{~m}$ and $601.5 \mathrm{~m}$, respectively. The other main simulation parameters are listed in Table I. In the simulation, we use the Peak function in MATLAB to simulate the terrain with the maximum height of $260 \mathrm{~m}$ and the maximum slope (in range) of $32.4^{\circ}$. The simulated terrain is shown in Figure. II and the simulating shift amounts in the azimuth and range directions are $-11.8 \mathrm{~m}$ and $5.3 \mathrm{~m}$, respectively.

Two pairs of SAR images corresponding to the two passes of the single-baseline InSAR system are generated through computer simulation. We use a real SAR image to generate the echo power of each SAR pixel. The SNR of the generated SAR images is $23 \mathrm{~dB}$. The statistical model is used to generate the three complex SAR images. The conventional methods are applied to generating two different interferograms which are shown in Figure.III(A) and Figure.III(B), respectively, from which we can see that the two interferograms are with different density due to the baseline length variation.
Figure.IV(A) is the correlation function of azimuth local frequency of the interferogram pair, and Figure.IV(B) is the local view of Figure.IV (A). Similarly, Figure.IV(C) is the correlation function of range local frequency of the interferogram pair, and Figure.IV(D) is the local view of Figure.IV(C). From the correlation functions of the azimuth and range frequencies of the interferogram pair, we can identify the peak positions clearly and determine the twodimensional offsets to be $-12 \mathrm{~m}$ (in azimuth direction) and $5 \mathrm{~m}$ (in range direction), respectively. Furthermore, the interpolation algorithms are applied to the correlation function and the two-dimensional shift amounts of subpixel level is obtained to be $-11.80 \mathrm{~m}$ and $5.29 \mathrm{~m}$, respectively, which are consistent with the simulation.

From the simulation results above, it can be seen that the accurate azimuth and range shift amounts are determined through the correlation of the local frequency of interferogram pairs. Finally, the pair of interferograms are aligned accurately via the resampling operation.

\section{CONCLUSION}

In practice, one of the most feasible ways to acquire multibaseline interferometric data is to observe the same ground scene repeatedly with a single-baseline InSAR system. For this system, this paper presents an innovative method for resolving the problem that repeat-pass SAR images are difficult to align due to the inherent decorrelation between repeat passes. The proposed method takes full advantage of the local frequencies of interferometric pair to determine the two-dimensional shift amounts in order that the interferograms can be accurately aligned, which is the indispensable precondition of subsequent processing steps. The method can effectively avoid the errors that are possibly introduced by two dimensional phase unwrapping. Finally, computer simulation demonstrated that it has the ability to achieve fine alignment of interferograms from single-baseline InSAR systems.

\section{ACKNOWLEDGMENT}

This work is supported by the National Natural Science Foundation of China (NSFC) under Grant 41301450, the Fundamental Research Funds for the Central Universities (QN2013066) and Exclusive Talent Funds of Northwest A\&F University (Z111021302).

\section{REFERENCES}

[1] P. A. Rosen, S. Hensley, I. R. Joughin, F. K. Li, S. N. Madsen, E. Rodriguesz, and R. M. Goldstein, "Synthetic aperture radar interferometry,” Proc. IEEE, Vol.88, No.3, pp333-382, 2000.

[2] R. Bamler and P. Hartl, "Synthetic aperture radar interferometry," Inv. Probl., Vol.14, No.1, ppR1-R54, 1998.

[3] A. Reigber and A. Moreira, "First demonstration of airborne SAR tomography using multibaseline L-band data," IEEE Trans. Geosci. Remote Sens., Vol.38, No.5, pp2142-2152, 2000.

[4] A. Ferretti, C. Prati, and F. Rocca, "Multibaseline InSAR DEM reconstruction: The wavelet approach," IEEE Trans. Geosci. Remote Sens., Vol.37, No.2, pp705-715, 1999.

[5] L. Ying, D. C. Munson, R. Koetter, and B. J. Frey, "Multibaseline InSAR terrain elevation estimation: A dynamic programming approach," in Proc. IEEE Int. Conf. Image Process., pp3202-3205, 2003.

[6] F. Gini and F. Lombardini, "Multibaseline cross-track SAR interferometry: a signal processing perspective,” IEEE Trans. on AES, 
Vol.20, No.8, pp71-93, 2005.

[7] F. Gini, F. Lombardini, and M. Montanari, “Layover solution in multibaseline SAR interferometry,” IEEE Trans. on AES, Vol.38, No.4, pp1344-1356, 2002.

[8] Wei. Xu, Ee. Chien. Chang, et al, "Phase unwrapping of SAR interferogram with multi-frequency or multi-baseline,” IGARSS'94, pp730-732, 1994.

[9] G. Ferraluolo, F. Meglio, et al, "DEM reconstruction accuracy in multichannel SAR interferometry,” IEEE Trans. Geosci. Remote Sens., Vol.47, No.1, pp191-201, 2009.

[10] G. Krieger, "TanDEM-X: Mission Performance and Product Definition,” First TanDEM-X Science Team Meeting, Dresden, 15, May, 2006.

[11] R. Scheiber and A. Moreira, "Coregistration of interferometric SAR images using spectral diversity,” IEEE. Trans. Geosci. Remote Sens., Vol.38, No.5, pp2179-2191, 2000.

[12] G. Fornaro and G. Franceschetti, "Image registration in interferometric SAR processing,” IEE Proc-Radar, Sonar Navig., Vol.142, No.6, 1995.

[13] D. C. Ghiglia and M. D. Pritt, Two Dimensional Phase Unwrapping, New York: Wiley, 1998.

[14] Hai Li, Zhenfang Li, Guisheng Liao, and Zheng Bao, “An Estimation Method for InSAR Interferometric Phase Combined with Image AutoCoregistration,” Science in China, Series F, Vol.49, No.3, pp386-396, 2006.

[15] P. Stoica, and A. Nehorai, "MUSIC, Maximum Likelihood, and Cramer-Rao Bound. IEEE Trans. on Acoust,” Speech Signal Process, Vol.37, No.5, pp720-741, 1989.

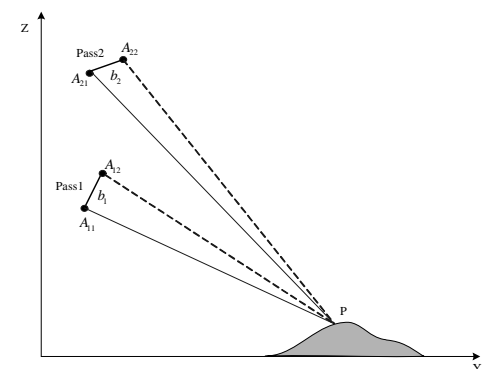

FIGURE I. GEOMETRY OF TWO PASSES OF A SINGLE-BASELINE INSAR SYSTEM.

TABLE I. MAIN SIMULATION PARAMETERS

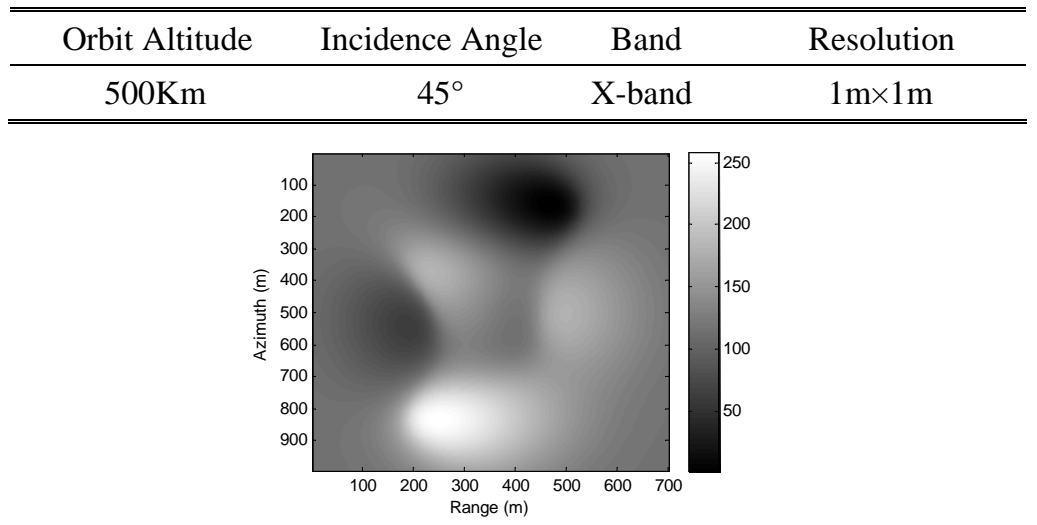

FIGURE II. SIMULATED TERRAIN.

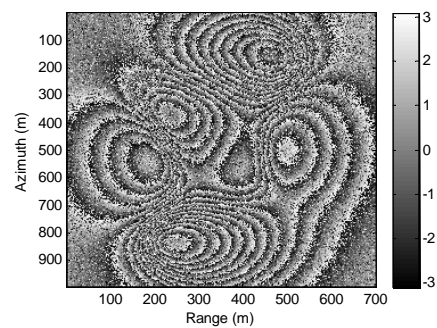

(A)

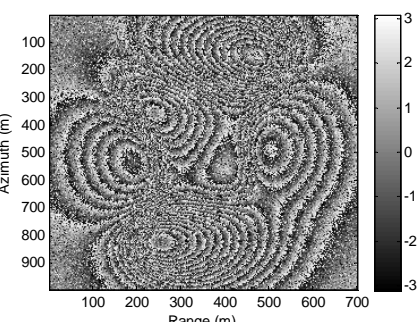

(B)

FIGURE III. INTERFEROGRAM PAIR OBTAINED BY THE TWO PASSES: (A) INTERFEROGRAM OF PASS1; (B) INTERFEROGRAM OF PASS2. 


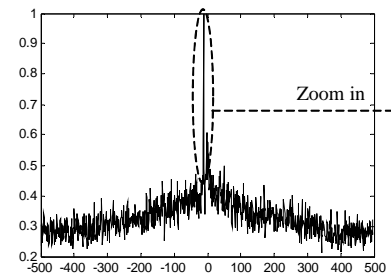

(A)

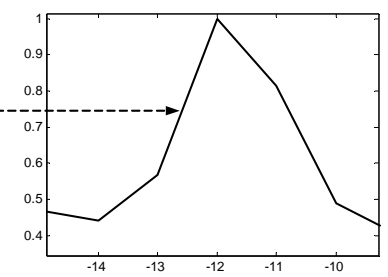

(B)

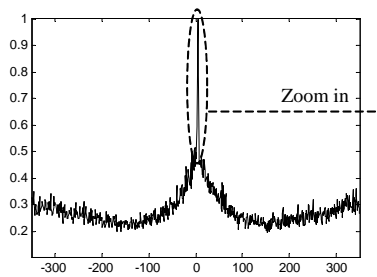

(C)

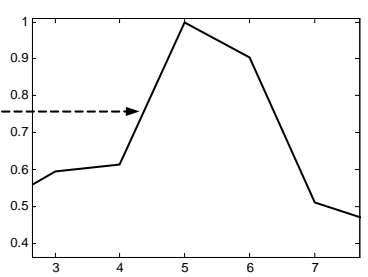

(D)

FIGURE IV. CORRELATION FUNCTIONS OF THE LOCAL FREQUENCIES: (A) CORRELATION FUNCTION OF AZIMUTH FREQUENCY;(B) LOCAL VIEW OF (A); (C) CORRELATION FUNCTION OF RANGE FREQUENCY; (D) LOCAL VIEW OF (C). 